16 | 2021

Simone et André Schwarz-Bart : nouvelles approches de l'oeuvre

\title{
Empathie mémorielle et transferts littéraires
}

\section{Nicole Lapierre}

\section{(2) OpenEdition}

\section{Journals}

Édition électronique

URL : https://journals.openedition.org/coma/5587

DOI : $10.4000 /$ coma.5587

ISSN : 2275-1742

\section{Éditeur}

Institut des textes \& manuscrits modernes (ITEM)

Référence électronique

Nicole Lapierre, «Empathie mémorielle et transferts littéraires », Continents manuscrits [En ligne], 16 | 2021, mis en ligne le 15 avril 2021, consulté le 10 septembre 2021. URL : http:// journals.openedition.org/coma/5587 ; DOI : https://doi.org/10.4000/coma.5587

Ce document a été généré automatiquement le 10 septembre 2021.

\section{(c)}

Continents manuscrits - Génétique des textes littéraires - Afrique, Caraîbe, dispora est mis à disposition selon les termes de la licence Creative Commons Attribution - Pas d'Utilisation Commerciale - Pas de Modification 4.0 International. 


\title{
Empathie mémorielle et transferts littéraires
}

\author{
Nicole Lapierre
}

1 Il me faut préciser d'entrée que je ne suis pas compétente en génétique des textes. Je ne viens même pas des études littéraires et, pour aggraver mon cas, je ne suis pas une spécialiste des œuvres d'André et de Simone Schwarz-Bart, comme Jean-Pierre Orban ou Francine Kaufmann. Alors à quel titre intervenir aujourd'hui, «d'où je parle » comme on disait dans les années 1970 ? En tant que lectrice, bien sûr, comme toutes et tous ici. Mais aussi en tant que socio-anthropologue ayant beaucoup travaillé sur les questions mémorielles et ayant obstinément tenté de déconstruire, de livre en livre, ce que j'appelle «la logique des places » : chacun sa culture, chacun son identité, chacun son territoire, chacun son histoire, chacun sa mémoire et les places, comme les vaches sont bien gardées.

2 Je pense qu'une mémoire de préjudices et de persécutions ne doit pas inévitablement en supplanter une autre. Il me semble important, au contraire, de rapprocher ces mémoires sans les trahir, ni les mettre en compétition. Il est fécond de les penser ou de les représenter ensemble, sans les hiérarchiser et sans réduire ou nier pour autant la singularité de chacune d'entre elles. C'est ce qui m'a conduite à la recherche aboutissant au livre intitulé Causes communes ${ }^{1}$. Un livre sur les rencontres, les alliances, les solidarités, les pensées et les mémoires croisées entre des Juifs et des Noirs (et non pas les Juifs et les Noirs, car ils ne forment pas des ensemble homogènes). Ce travail s'inscrivait dans le prolongement de mes travaux précédents ${ }^{2}$, mais il répondait aussi à ce que je ressentais comme une urgence du temps: combattre ce que l'on appelle, improprement d'ailleurs, la "concurrence des victimes", à laquelle l'humoriste Dieudonné donnait alors une forte et néfaste visibilité. C'est à l'occasion de ce travail que j'ai retrouvé André et Simone Schwarz-Bart. Je dis «retrouvé » car j'avais eu la chance de rencontrer André bien avant, en compagnie d'un de ses vieux amis, le peintre Isaac Cielniker, que je connaissais aussi. Quant à Simone, nous nous sommes vues à plusieurs reprises chez nos amis communs, Sylvie et Édouard Glissant, et elle a eu la générosité de m'accorder un long entretien quand je préparais Causes communes. 
Elle y racontait leur rencontre, leur amour, la personnalité d'André et de ses amis, la façon dont progressivement leurs mémoires se sont entremêlées jusque dans l'écriture. Toutes choses qu'elle a depuis racontées de façon beaucoup plus détaillée dans son livre réalisé avec Yann Plougastel Nous n'avons pas vu passer les jours ${ }^{3}$.

3 Voici un fragment de ce qu'elle me disait alors :

Après Le Dernier des Justes, il y avait une telle pression et une telle attente qu'il perpétue cette même veine, cette même inspiration, qu'il a peut-être voulu « casser son image en mille morceaux » comme il le dit dans certains textes. D'abord avec ce Plat de porc, quelque chose de totalement impie. Je pense qu'il a en même temps voulu montrer que c'était cela aussi pour lui être juif : avoir la préoccupation d'autres mondes. Ne pas seulement faire comme la cigogne qui ne s'occupe que des siens. D'ailleurs, il s'est toujours intéressé au monde et pas seulement à son monde, dans ses amitiés comme en littérature. Mais c'est difficile, c'est un chemin solitaire où l'on se fait caillasser de tous les côtés : du côté d'où l'on est, d'où l'on vient, mais aussi par ceux-là même que vous portez. Et cela vous met complètement en porte à faux, cela vous éclate. Il faut avoir la force de vivre l'inconfort que cela génère. C'était très douloureux.

Fig. 1 : Couverture originale d'Un plat de porc aux bananes vertes, 1967

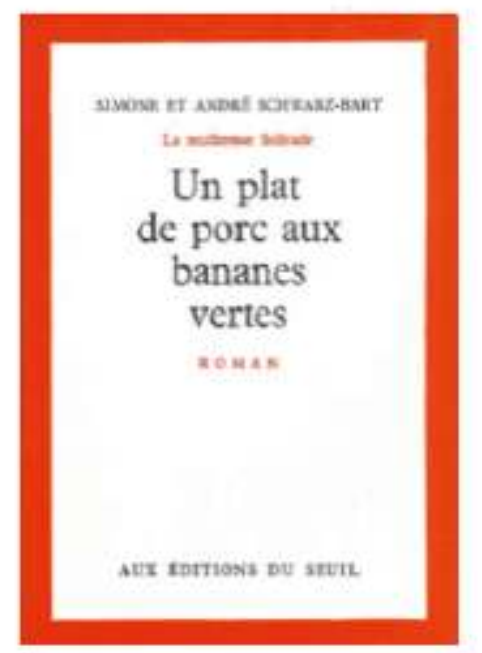

4 Je voudrais revenir sur ce livre, Un plat de porc aux bananes vertes, sur son inspiration, son propos, son écriture et sa réception. Simone et André le publient sous leurs deux noms, au Seuil, début $1967^{4}$. Il est dédié conjointement à Aimé Césaire, chantre de la négritude et à Elie Wiesel, écrivain témoin de la souffrance juive. Et il se présente comme le journal en sept cahiers d'une vieille Martiniquaise. Mariotte - c'est son nom finit ses jours dans le « Trou ", un sordide hospice de vieillards à Paris. Elle songe à son île, aux histoires de son enfance, aux proches qu'elle a perdus, à sa petite-fille la Unetelle qui s'est suicidée, à la trace lointaine d'une ancêtre insoumise du nom de Solitude et à ce mets délicieux, le plat de porc aux bananes vertes, qu'elle ne mangera plus. Malgré sa vue qui baisse, le regard qu'elle porte autour d'elle est d'une lucidité impitoyable. Elle voit tout, l'ordinaire raciste, le dégoût ou la concupiscence qu'elle suscite, la lubricité minable de Monsieur Nicolo, le concierge, qui la fait danser et chanter "Cé l'amou Doudou» pour un permis de sortie, la décrépitude de ses compagnes d'infortune et sa propre déchéance. Dans ce lieu de relégation peuplé de 
créatures misérables, les pensionnaires se chamaillent pour un peu de nourriture, s'accrochent à des lambeaux de plaisir ou se laissent lentement couler.

5 Ainsi, l'une d'elles, à la conscience vacillante, est hantée par un passé qui n'est peutêtre pas le sien. En proie à un mal mystérieux, elle se jette de toutes ses forces, la tête la première, contre le mur, ou elle se pique jusqu'au sang un matricule imaginaire sur l'avant-bras gauche, chaque fois qu'elle entend le mot «juif». Mariotte se demande quels démons habitent cette pauvre démente nommée Louise Duployé. Son comportement est énigmatique. Elle n'a pas été déportée. Elle n'est sans doute pas juive. Mais, pour une raison inexpliquée, elle a été marquée par la catastrophe au point de vouloir, de façon obsessionnelle et compulsive, l'inscrire dans sa chair. Peut-être s'agit-il des accès maniaques d'une survivante traumatisée ? Peut-être s'agit-il d'une identification délirante? Je ne peux m'empêcher d'y voir, pour ma part, une anticipation de la façon dont certains vont s'identifier et s'inventer une histoire juive afin d'inscrire leur souffrance - d'orphelin, de persécuté, de minoritaire opprimé dans la référence cardinale du malheur que deviendra la Shoah. En témoignent des récits singuliers, parfois des livres à succès où l'identification a été poussée jusqu'à la mystification ${ }^{5}$, ou encore des revendications généalogiques comme celles des Black Jews américains ${ }^{6}$.

6 Si saisissant soit-il, ce personnage de Louise est néanmoins secondaire. C'est loin d'être la seule allusion au génocide dans le livre. Il y a celles et ceux qui voudraient en finir « comme les youpins : une piqûre et hop!» (p. 76). Ou Madame Peuchemard qui, un jour, déclare, constatant qu'il reste encore beaucoup de Juifs : «à ce qu'il semble leurs crématoires, c'étaient des couveuses ?..." " (p. 77). Ce passé-là rôde dans le présent de l'hospice, comme les cauchemars qui font parfois hurler Mariotte. Mais au-delà de ces passages qui s'y réfèrent directement, c'est tout le roman qui opère un déplacement permanent de la mémoire juive sous-jacente à la mémoire noire. Et de l'une et l'autre à la condition de la vieillesse abandonnée. Une condition plus dure encore s'agissant des femmes. Comme les Juifs pendant la guerre, les Noirs et les vieux sont bons pour la relégation ou la mort... L'hospice est une parodie de camp de concentration où l'on se bat pour un quignon de pain, où règne l'arbitraire et où la déshumanisation ronge les pensionnaires. C'est une institution totalitaire au sens donné à ce terme par le sociologue Erving Goffmann ${ }^{7}$.

7 Mariotte, enfant, a réchappé à l'éruption de la Montagne Pelée qui a emporté sa mère et englouti toute la ville de Saint-Pierre en Martinique le 8 mai 1902. Tout un monde là aussi a été détruit, enfoui sous les cendres. L'enfant survivant, le dernier témoin à qui incombe la charge du récit, est évidemment un topos des histoires de catastrophes, qu'elles soient d'origine naturelle ou dues à la cruauté humaine. C'est également le rôle du narrateur qu'André Schwarz-Bart, orphelin survivant, s'était donné dans son premier ouvrage, Le Dernier des Justes. Rôle qu'il délègue cette fois à la vieille Martiniquaise.

8 Dans ce roman, une histoire s'inscrit ainsi dans l'intrigue d'une autre. L'imaginaire d'André Schwarz-Bart créant Mariotte et ses cahiers, est profondément pétri de ce qu'il a appris sur les camps, de ce qu'il a vécu de la période de la collaboration en France, mais aussi de ce qu'il connaît de l'histoire antillaise et de ce qu'il sait du racisme banalisé. Le "Trou », c'est tout cela à la fois : l'humanité dégradée, vouée à la mort, le chantage minable du concierge, l'obscénité de Monsieur Moreau émoustillé par des souvenirs de bordées tropicales, ou encore l'hypocrisie bien-pensante, l'indifférence au 
malheur, la dégradation des êtres. Un plat de porc aux bananes vertes entrecroise une peinture expressionniste au trait acéré et un émouvant récit intime. C'est un livre à la fois rageur et infiniment triste.

9 J'ai parlé à ce propos de "l'imaginaire d'André » car, selon Simone, il est bien l'auteur principal de ce livre et y a mis beaucoup de ses propres obsessions. Permettez-moi de la citer encore une fois :

Il faut savoir que toute la trame c'est lui. Cet asile, ces personnages, c'est lui. Il y a des notes où il dit «Mariotte, c'est moi, Louise, c'est moi »... Je ne pourrais pas rentrer dans des personnages comme ceux-là, c'est impossible pour moi, c'est trop dur. Je ne pourrais pas aller chercher dans cette fange-là sans me noyer. Lui pouvait refaire surface et respirer comme un poisson qui sort des abysses, moi je vais me noyer. Il écrivait cela à partir de l'univers concentrationnaire. C'était le noyau même de toutes ces situations. Je suis intervenue au niveau du langage, quelquefois. Au niveau du langage, c'est tout. Je ne voulais pas signer, car je ne pensais pas avoir suffisamment amené de moi-même. Mais il m'a dit «si tu ne signes pas avec moi, cela ne paraîtra pas ", alors j'ai accepté. Je pensais que cela devait paraître. ${ }^{8}$

10 À sa sortie, le roman fut mal reçu par la critique et boudé par les lecteurs. Sans doute en raison de cette hybridité ou de son côté trop grinçant, trop féroce. Car sa peinture de la vieillesse est cruelle, voire choquante. Les victimes ne sont ni touchantes, ni belles. Les bonnes œuvres font l'objet de railleries, comme le dévouement de sœur Marie des Anges transportant les pots de chambre malodorants car, ironise Mariotte: elle «nous aime de toutes les forces que dispense au sauvage chrétien l'envie de faire son salut » (p. 18). Quant à Mariotte, si elle a jamais eu la foi, elle l'a perdue : «Je crois, écrit-elle, en ces vieux murs suintants où les larmes de la pierre ont creusé des lits dérisoires dans le plâtre jauni. Je crois au réfectoire où nous retrouvons les hommes du pavillon B sous la bénédiction du Christ d'un quintal qui saigne midi et soir dans notre soupe, sans réussir à lui donner un goût de viande. » (Ibid.) Cet univers et cette dérision iconoclaste ne correspondent pas à ce que l'on attendait de l'auteur du Dernier des justes. Livre auquel, d'ailleurs, certains avaient reproché d'être trop «lacrymal» ou trop christique. André Schwarz-Bart n'est pas où on l'attendait. Pour l'auteur d'un «bestseller juif », même le titre, si peu casher, semble défier les attentes des lecteurs.

Le style est complètement différent et la personnalité de l'auteur également, mais l'humour noir, l'ironie, la dérision travestissant la détresse des Juifs rescapés et des Noirs américains me font penser à Tulipe de Romain Gary ${ }^{9}$. Un autre livre intempestif, hors cadre, d'un écrivain qui, lui aussi, ne voulait pas être assigné au rôle et à l'écriture que l'on attendait de lui. Ni comme Compagnon de la Libération - publié en 1946, Tulipe ironise sur la morale des vainqueurs -, ni plus tard comme écrivain à succès ayant décroché le prix Goncourt. Et l'on sait qu'il rejouera la donne avec Émile Ajar et ce personnage de Rosa dans La Vie devant soi, rescapée d'Auschwitz, ancienne prostituée qui accueille les gamins perdus.

12 Le roman mêlé, dérangeant, provocateur sur fond de désespoir, dans lequel André Schwarz-Bart opère une translation entre les thèmes juifs et noirs, est tout autant désaccordé et intempestif. C'est pour cela sans doute qu'il a été souvent relégué, voire oublié, dans les analyses des œuvres de l'auteur. Certains commentateurs ont parlé d'un ouvrage " décousu par sa structure elliptique ${ }^{10}$ ». Or c'est un texte audacieux, dans sa composition comme dans sa verve ravageuse. Le mélange des langues - le français élaboré de Mariotte, le style haché et relâché de l'hospice, les mots de créole (la touche de Simone), et la construction fragmentée préfigurent les écritures composites d'une 
certaine postmodernité qui cherchent à réfracter la collision des temps et l'hybridation des sociétés.

13 Écrire à contretemps et être lu à contresens fut une expérience douloureuse pour André Schwarz-Bart. Il avait publié Le Dernier des Justes en 1959 quand la mémoire de la Shoah n'était pas encore célébrée (le mot lui-même n'allait s'imposer que bien plus tard avec le film de Claude Lanzmann) et alors que dans le monde juif une vision plus combattante prévalait, chez les communistes comme chez les sionistes. Il a écrit (ou coécrit) Un plat de porc aux bananes vertes dans un mélange des thèmes et des souffrances qui n'était guère en phase avec le fond de l'air vif et léger de la fin des années 1960. En outre, ce livre ne pouvait que décevoir ceux qui attendaient un roman juif, ou tout autre genre bien défini d'ailleurs. Certains ont voulu y voir un roman exclusivement antillais écrit par une Antillaise, Simone, que son mari aurait exploitée en cosignant. Le nom de ce dernier a même parfois été effacé. ${ }^{11}$

Pour André, ce livre devait être le début d'un grand cycle romanesque en sept volumes dont l'histoire s'étendrait sur deux siècles, de 1760 aux années 1950 et, chemin faisant, croiserait des personnages noirs et juifs. Comme vous le savez, seul le premier, $L a$ Mulâtresse Solitude (titre qui devait être celui de l'ensemble), fut publié en 1972. Ce roman, inspiré d'un personnage réel et largement oublié, a véritablement créé une haute figure de la mémoire guadeloupéenne. Une imposante statue de Solitude, la négresse marron, enceinte, héroïque et rebelle, a d'ailleurs été érigée à Pointe-à-Pitre en 1999. Une histoire antillaise, certes, et pourtant... À la fin du roman, André SchwarzBart imagine un étranger qui lui ressemble, visitant les ruines de l'ancienne habitation Danglemont à Matouba (l'endroit où le révolutionnaire Louis Delgrès, ses trois cents camarades et parmi eux Solitude ont préféré se faire sauter plutôt que de se rendre). Et l'écrivain termine sur ces mots :

Alors, s'il tient à saluer une mémoire, il emplira l'espace environnant de son imagination; et si le sort lui est favorable, toutes sortes de figures humaines se dresseront autour de lui, comme font encore, dit-on, sous les yeux d'autres voyageurs, les fantômes qui errent parmi les ruines humiliées du Ghetto de Varsovie (p. 156).

Quoiqu'il en soit, dans le contexte d'affirmation identitaire et de revendication mémorielle antillaises, certains lui ont dénié le droit d'écrire un tel roman. Ils ont contesté sa légitimité ou sa capacité à restituer une histoire qui n'était pas la sienne. Il y a eu des interrogations sur la paternité du texte, attribuant l'essentiel, voire la totalité de celle-ci à Simone. Plus tard, de façon plus rude encore, une critique simpliste se prétendant postcoloniale et une critique réductrice se réclamant du féminisme accuseront ce mâle blanc occidental de pillage culturel et de domination machiste. Aux yeux de ses accusateurs, il n'avait ni la bonne couleur de peau, ni le bon sexe, ni la bonne place. En dépit des encouragements d'Aimé Césaire, ces incompréhensions tenaces et ces accusations injustes l'ont blessé. Raison, ou une des raisons pour lesquelles il s'est arrêté, non pas d'écrire, mais de publier.

16 Michael Rothberg, dans un ouvrage publié aux États-Unis et récemment traduit ${ }^{12}$, salue cette entreprise littéraire qui cherche, écrit-il, à « développer une esthétique fondée sur l'hospitalité aux histoires de l'autre ». Mais il considère qu'André Schwarz-Bart n'a pas pu aller jusqu'au bout de cette démarche pionnière, faute d'avoir trouvé une forme narrative adéquate. C'est-à-dire une façon de raconter les désastres et les articuler, dans leur singularité, sans les réinscrire dans un récit classique. Selon lui, cet " échec » serait la cause du silence dans lequel s'est finalement enfermé l'auteur après la 
parution de La Mulâtresse Solitude. Le fait que, par la suite, ce dernier, insatisfait, ait sans relâche détruit une grande partie de ses manuscrits ultérieurs, corrobore en partie, mais en partie seulement, cette hypothèse sur le silence. Nul doute que la réception décevante, et parfois durement polémique de ses textes y ont également contribué. D'autant plus que cette difficulté à trouver une forme était surmontée dans l'écriture fragmentaire et transmémorielle d'Un plat de porc aux bananes vertes, le plus mal aimé et le moins étudié de ses livres. Ce que semble corroborer un passage de L'Étoile du matin, ce livre posthume retrouvé et reconstitué par Simone ${ }^{13}$. C'est l'histoire de Haïm, enfant au début de la guerre, qui traverse le pire, survit et se met à écrire. Haïm, y lit-on, " avait publié deux ou trois livres, autrefois, et maintenant il se vivait comme un schlemiel, un homme qui avait perdu son ombre. Mais il n'avait pas seulement perdu son ombre, il avait aussi perdu son moi et il était comme la Mulâtresse Solitude, l'héroïne d'un de ses romans, du temps où elle n'existait pas. Il était en deuil de la littérature, en deuil de lui-même» (p. 203). De ce double évident de Schwarz-Bart, il est dit encore : «Il avait cherché mille approches pour écrire sur cette planète Auschwitz, mais il n'était jamais arrivé au bout : chaque fois la honte d'écrire sur les morts l'avait emporté et il avait tout détruit.» (p. 205) Une de ces approches, pourtant, lui avait semblé presque aboutie, pensait-il en relisant Un plat de porc aux bananes vertes, mais les lecteurs n'avaient pas compris. Aussi peut-on dire, je crois, que son silence, ou plutôt son refus de lâcher ses manuscrits et de les publier, est tout autant celui d'un auteur qui a le sentiment désolant de ne pas être entendu ni lu comme il voudrait.

Michael Rothberg soulève une autre question sur les liens entre mémoire juive et mémoire noire dans l'œuvre, en s'appuyant sur un entretien accordé par Schwarz-Bart à un journal américain, Midstream, en mars 1967 (donc après la parution du Plat de porc). Questionné à ce sujet, il explique :

Il n'y a nullement séparation entre une ancienne inspiration et une autre, qui lui aurait succédé, car l'idée et la décision initiale d'écrire ce livre [Un plat de porc] remonte bien avant l'achèvement du Dernier des Justes : la décision date de 1955 et Le Dernier des Justes a été terminé en $1959 .{ }^{14}$

Il précise aussi dans que, dès le milieu des années 1950, il fréquentait des intellectuels antillais au Quartier latin et était frappé par la permanence du lointain passé de l'esclavage dans leur présent. Associer cette mémoire longue à celle des Juifs lui permettait, disait-il, d'arracher la seconde à la "solitude de la destinée juive", marquée par le passé proche du génocide. S'appuyant sur ces déclarations et le fait qu'en 1955 André Schwarz-Bart avait profondément remanié le manuscrit du Dernier des Justes en lui donnant une profondeur temporelle accrue, conduisent Michael Rothberg à affirmer que la mémoire de l'esclavage a permis de déployer la mémoire juive et son récit. Une analyse opposée à celle de Francine Kaufmann pour qui le cycle romanesque de La Mulâtresse Solitude «ne saurait se concevoir sans la mémoire de la Shoah qui ouvre le Juif à toute souffrance humaine ${ }^{15}$ ».

Il s'agit là d'une question importante, notamment du point de vue de la genèse de l'œuvre. Au delà, elle renvoie à deux lectures différentes. À travers les interactions de ce que Michael Rothberg nomme une "mémoire multidirectionnelle" (dont il donne des exemples chez plusieurs auteurs, notamment Charlotte Delbo), la réminiscence d'autres drames historiques a pu contribuer à l'évocation et à la reconnaissance de la spécificité du génocide dans l'espace public. Francine Kaufmann, quant à elle, insiste sur la centralité de la mémoire juive. De mon point de vue, la question essentielle est celle de la Relation entre les deux expériences, vue par Schwarz-Bart. Je parle ici de 
Relation, avec une majuscule, au sens où l'entend Édouard Glissant. Une Relation qui relie, relaie, relate ${ }^{16}$ les cultures, les mémoires et les hommes.

Glissant et Schwarz-Bart étaient ensemble avec Fanon au deuxième congrès des écrivains et artistes noirs, organisé par la revue Présence Africaine, au printemps 1959, à Rome. Dans un entretien, publié par Le Figaro littéraire en janvier 1967, le second expliquait :

En fait, depuis longtemps déjà je connaissais et fréquentais les milieux antillais de Paris. Et ce qui me portait vers eux, c'était tout autre chose qu'une froide solidarité avec nos frères de couleur, comme on dit maintenant. Non, il s'est agi d'une grande et vive sympathie que j'ai ressentie, presque aussitôt, pour leur façon d'être, pour leur gaité, leur douceur, leur sagesse, leur art de vivre, et pour cette espèce de lyrisme verbal qui fait que dans la bouche d'un Antillais, d'un vrai Antillais, tout devient poésie. ${ }^{17}$

Ce passage rappelle la critique $\mathrm{du}$ « fraternalisme » du grand frère communiste, dans la Lettre à Maurice Thorez d'Aimé Césaire, en 1956. André Schwarz-Bart refuse les discours d'émancipation convenus ou conseillés par un grand frère à la poigne ferme. Il ne parle pas de compassion non plus, mais du "pouvoir sans limite de la sympathie humaine", en précisant que «l'essence du dialogue n'est pas dans les idées universelles communes aux interlocuteurs ni dans les idées que l'un se fait de l'autre, mais dans la rencontre même, dans l'invocation, dans le pouvoir qu'a le Moi de dire $\mathrm{Tu}^{18}$ ».

En écho, on songe aux mots de Frantz Fanon qui affirmait dans Peaux noires, masques blancs: "L'antisémitisme me touche en pleine chair, je m'émeus, une contestation effroyable m'anémie, on me refuse la possibilité d'être un homme. ${ }^{19}$ » Et qui, dans le même livre, s'interrogeait : «Ma liberté ne m'est-elle pas donnée pour ériger le monde du toi $?^{20} »$

Fig. 2 : De gauche à droite, Albert Béville (alias Paul Niger, 1915-1962), Jacques Rabemananjara (1913-2005), Édouard Glissant (1928-2011), André Schwarz-Bart (1928-2006). Début des années soixante

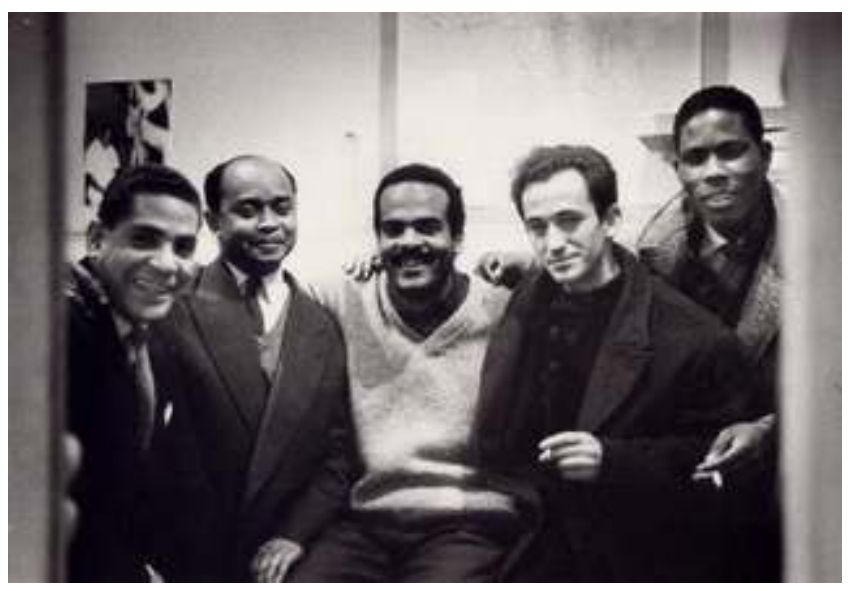

(c) www.edouardglissant.fr

On songe également à ceux d'Édouard Glissant, dans Une nouvelle région du monde :

Chacun de nous a besoin de la mémoire de l'autre, parce qu'il n'y va pas d'une vertu de compassion ou de charité, mais d'une lucidité nouvelle dans un processus de la Relation. Et si nous voulons partager la beauté du monde, si nous voulons être solidaires de ses souffrances, nous devons apprendre à nous souvenir ensemble. ${ }^{21}$ 
Tous évoquent ainsi l'empathie, qui est plus que la sympathie. C'est un état d'esprit, une disponibilité, une capacité à adopter un moment le point de vue de l'autre pour s'en trouver enrichi et transformé. «Tu échanges, changeant avec l'autre, sans pour autant te perdre ni te dénaturer ${ }^{22}$ ", répète ainsi à l'envi Édouard Glissant. C'est cette inspiration et cette empathie qui favorisent les transferts littéraires d'un univers à l'autre. Des transferts qui permettent de dire par un détour, quand il n'y a pas de retour possible.

\section{NOTES}

1. Nicole Lapierre, Causes communes, Paris, Stock, 2011.

2. Nicole Lapierre, Pensons ailleurs, Paris, Stock, 2004, paru en coll. « Folio Essais » en 2006.

3. Simone Schwarz-Bart et Yann Plougastel, Nous n'avons pas vu passer les jours, Paris, Grasset, 2019.

4. Simone et André Schwarz-Bart, Un plat de porc aux bananes vertes [1967], Paris, Seuil, coll. « Points », 2003 (la pagination dans cet article renvoie à cette réédition).

5. Binjamin Wilkomirski, Fragments. Une enfance. 1939-1948, Paris, Calmann-Lévy, 1997 (traduit de l'édition allemande de 1995) et Misha de Fonseca, Survivre avec les loups, Paris, Robert Laffont, 1997.

6. Voir Nicole Lapierre, Causes communes, op. cit., chap. 5 « Peau noires, masque juif », p. 235-262.

7. Erving Goffmann, Asiles. Étude sur la condition sociale des malades mentaux, Paris, Éditions de Minuit, 1975.

8. Entretien avec l'auteure, voir supra.

9. Romain Gary, Tulipe, Paris, Gallimard, coll. « Folio », 2010.

10. Kathleen Gyssels, Filles de Solitude. Essai sur les identités antillaises dans les (auto)biographies fictives de Simone et André Schwarz-Bart, Paris, L'Harmattan, 1996, p. 22.

11. Par exemple dans le catalogue de l'exposition "Présence Africaine», au Musée du quai Branly, Gradhiva, n 10, numéro spécial, 2009, p. 235.

12. Michael Rothberg, Mémoire multidirectionnelle. Repenser l'Holocauste à l'aune de la décolonisation, Paris, Éd. Petra, 2018.

13. André Schwarz-Bart, L'Étoile du matin, Paris, Seuil, 2009.

14. Michel Salomon, «Jewishness and Negritude: An Interview with André Schwarz-Bart», Midstream, mars 1967, cité par Michael Rothberg, op. cit., p. 142.

15. Francine Kaufmann, «Les enjeux de la polémique autour du premier best-seller français de la littérature de la Shoah », Myriam Ruszniewski-Dahan et Georges Bensoussan (éd.), Revue d'Histoire de la Shoah, $\mathrm{n}^{\circ} 76$, septembre-décembre 2002, note 20, p. 96.

16. Édouard Glissant, Poétique de la Relation, Paris, Gallimard, 1991, p. 187.

17. André Schwarz-Bart, "Pourquoi j'ai écrit La Mulâtresse Solitude », Figaro littéraire, n 1084, 26 janvier 1967, p. 1.

18. Ibid., p. 8.

19. Frantz Fanon, Peau noire, masques blancs [1952], Paris, Seuil, coll. « Points », 1971, p. 170.

20. Ibid, p. 224.

21. Édouard Glissant, Une nouvelle région du monde, Paris, Gallimard, 2006, p. 161.

22. Édouard Glissant, La Cohée du Lamentin. Poétique V, Paris, Gallimard, 2005, p. 38. 


\section{RÉSUMÉS}

L'exposé (Séminaire Schwarz-Bart, février 2020) converti ici en article, s'appuie, notamment, sur l'ouvrage Causes Communes, Des Juifs et des Noirs (Stock, 2011). Il traite du transfert mémoriel dans le parcours et l'œuvre d'André Schwarz-Bart, en particulier à travers le roman Un plat de porc aux bananes vertes signé par André et Simone Schwarz-Bart (Seuil, 1967). S'appuyant sur le concept de «mémoire multidirectionnelle » de Michael Rothberg et celui de « Relation » d'Édouard Glissant, l'auteure de l'article voit dans l'approche d'André Schwarz-Bart de l'esclavage des Noirs, plutôt qu'une capacité de l'appréhender à partir du drame qui serait central de la Shoah, celle du pouvoir de rencontrer l'Autre, du « Moi à dire Tu ».

Nicole Lapierre's presentation at the Schwarz-Bart Seminar of February 2020, which this paper is based on, draws on the book Causes Communes, Des Juifs et des Noirs (Stock, 2011). It addresses the transfer of memory through the work of André Schwarz-Bart, in particular in the novel Un plat de porc aux bananes vertes by André and Simone Schwarz-Bart (Seuil, 1967). Drawing on Michael Rothberg's concept of "multidirectional memory" and Édouard Glissant's concept of "Relation", the author of the article sees, in André Schwarz-Bart's exploration of Black slavery, the power to meet the Other, of the «Me to say You», rather than an approach of the tragedy from a Shoahcentric perspective.

\section{INDEX}

Keywords : Jewish memory, Black memory, Literary transfer, Memory transfer, Shoah, Slavery, Édouard Glissant, Frantz Fanon, Old age, Romain Gary, Empathy, Relationship

Mots-clés : mémoire juive, mémoire noire, transfert mémoriel, transfert littéraire, Shoah, esclavage, vieillesse, Édouard Glissant, Frantz Fanon, Romain Gary, empathie, relation

\section{AUTEUR}

\section{NICOLE LAPIERRE}

NICOLE LAPIERRE, socio-anthropologue, est directrice de recherche émérite au CNRS. Ses sujets de recherche sont : les relations entre générations ; les identités et la nomination; le thème de l'étranger dans les sciences sociales ; les migrations, les minorités et la mémoire. Elle est auteur ou co-auteur d'une douzaine d'ouvrages de référence en sciences sociales et a dirigé quatre ouvrages collectifs. Son ouvrage, Sauve qui peut la vie, a obtenu le prix Médicis essai en 2015. Son dernier livre, Faut-il se ressembler pour s'assembler ? est paru au Seuil en 2020. Elle codirige la revue Communications et dirige la collection « Un ordre d'idées » chez Stock. http://www.iiac.cnrs.fr/ article22.html 